Open Peer Review on Qeios

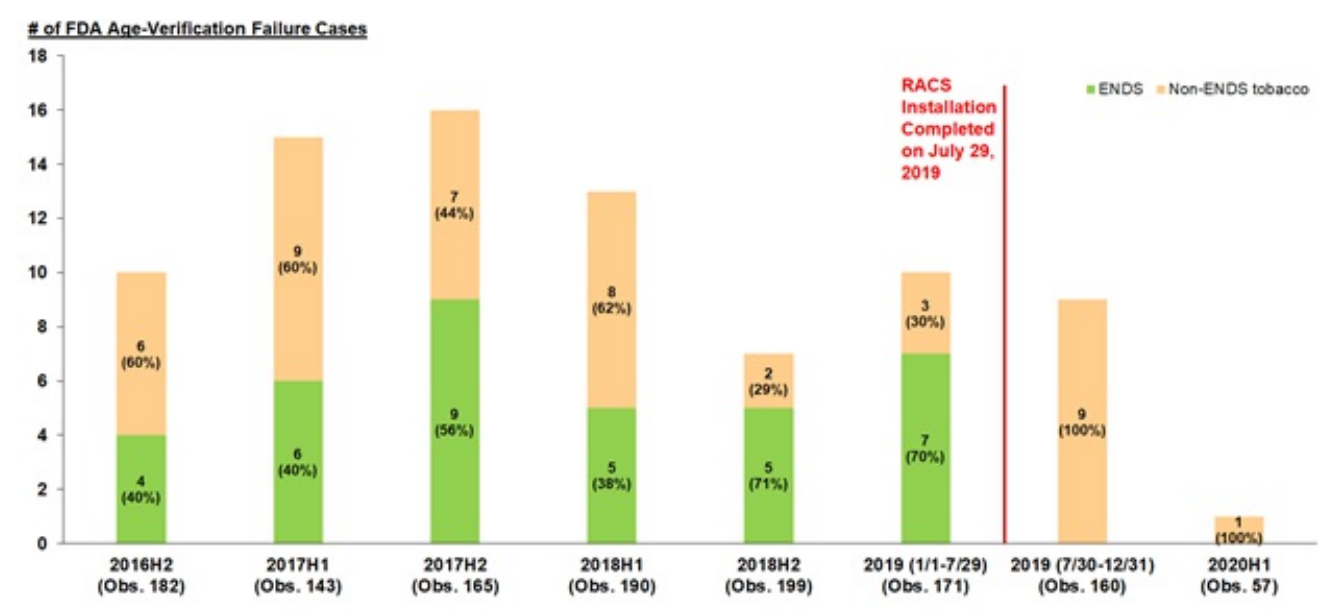

\title{
Evidence from FDA Compliance Check Inspections on the Effectiveness of Retail Access Controls on Tobacco Purchases
}

Tengjiao Chen, Adam Zion ${ }^{1}$, Jonah Joselow ${ }^{1}$, Parker Kasmer ${ }^{1}$

1 JUUL Labs

Funding: Funding for this study was provided by JUUL Labs Inc.

Potential competing interests: All of the authors are full-time employees of JUUL Labs, Inc. The authors contributed to writing and review of the paper and had access to the data. The sponsor approved the research plan and provided comment on a near-final draft of the paper.

\section{Abstract}

This study analyzes data from FDA's compliance check inspections of tobacco product retailers to assess the effectiveness of Retail Access Control Standards ${ }^{\mathrm{TM}}$ (RACS). RACS is a standards-based technology solution at the point-of-sale that automatically prompts electronic ID scanning to verify the age of the purchaser of electronic nicotine delivery systems (ENDS) and imposes automated product-quantity purchase limits (to reduce the potential for social sourcing). Supported by JUUL Labs, Inc., RACS is being adopted for the sale of JUUL products, but has not uniformly been implemented for other tobacco products. This study assesses the incidence of FDA compliance check inspection failures across tobaccoproduct types at a regional chain of convenience stores that implemented RACS, and compares it to those observed at similar retail locations without RACS. At the chain that implemented RACS, in the 8 months after RACS was installed, there were a total of 10 inspection failures, of which none were ENDSrelated. At the comparator sites, $28 \%$ of failures were ENDS-related. These findings provide real-world evidence that an automated, technologically-based solution for age-verification at the point-of-sale, such as RACS, may effectively restrict underage access and thus contribute to reducing underage use. This 
points to the potential benefit of adopting similar approaches across all tobacco products and potentially other age-restricted products.

Keywords: Retail Access Control Standards (RACS); FDA compliance check inspections; age verification; youth prevention; electronic nicotine delivery system (ENDS); enhanced access controls; tobacco.

\section{Introduction}

Those who are underage should not use tobacco products, including electronic nicotine delivery systems (ENDS). Reducing underage access is a top priority for regulators, policymakers, public health, and industry, including JUUL Labs, Inc. (JLI). The U.S. Food and Drug Administration (FDA) has recommended that tobacco product manufacturers and retailers adopt additional safeguards and mitigation strategies to better restrict underage access and improve retailer compliance with age-verification requirements. ${ }^{[1]}$ Such strategies could include establishing or enhancing programs to monitor retailer compliance with age-verification and sales restrictions and limiting the quantity of products that a customer may purchase within a given time period.

As part of its commitment to combat underage use, JLI has developed and fielded a retailer trade program, called Retail Access Control Standards ${ }^{\mathrm{TM}}$ (RACS), which aims to restrict underage access to JUUL products at the point-of-sale (POS). RACS was developed as a software-based standards program for retailers and software vendors to adopt within pre-existing POS systems or acquire through new POS system technology. In 2019, JLI began working with major POS vendors, technology providers, and retailers with proprietary POS systems to develop and implement the technical components for RACS. JLI did not provide or create proprietary software, code or hardware, but rather prepared the rules for complying with RACS for adaptation into POS systems. JLI then worked with retailers and POS system providers to encourage adoption of RACS for the sale of JUUL products. Since 2019, 26 POS systems, servicing 70,000 retail outlets in the United States, have developed software based on these standards.

RACS automates transactions requiring age-verification, ensuring that the retailer automatically verifies the age of the purchaser by pausing a purchase until an ID is scanned and by automatically restricting the amount of product that can be purchased. A RACS compliant POS begins by identifying purchases that include JUUL products. ${ }^{[2]}$ Whenever a transaction contains these products, the POS will (i) require ID scanning to verify the age and ID expiration; (ii) limit the amount of product that can be purchased to 1 device and/or 4 JUULpod packages (or for any manufacturer-specified limit); and (iii) limit the manual override by the retail clerk so that avoiding ID scanning or completing purchases that exceed the specified limits is not possible with manager-level approval.

While originally designed for use with JUUL products, the features were developed to be product-agnostic, so a retailer could choose to apply the restrictions to other age-restricted products such as non-JUUL ENDS, 
non-ENDS tobacco, alcohol or caffeinated drinks. JLI conducted a pilot study of its RACS program for JUUL products across a subset of 3 chain retailers selling tobacco products in 3 states. ${ }^{[3]}$ Results from the pilot demonstrated that failure rates of mystery-shopper audits for age verification and compliance with product-quantity limits declined to near zero for purchases involving JUUL products after RACS was implemented at the POS. ${ }^{[3]}$

This study uses publicly-available government data to assess the effectiveness of the RACS program in a multi-state chain retailer. Data comes from FDA's compliance check program to evaluate retailer compliance with tobacco product legal purchase age restrictions. The FDA conducts compliance check inspections of tobacco product retailers to assess adherence with federal tobacco product purchase ageverification requirements. ${ }^{[4]}$ In these inspections, the FDA uses shoppers, who are under the minimum purchasing age for tobacco products, to determine whether the retailer has adhered with federal ageverification requirements. These minors, trained for the purpose and supervised by FDA inspectors, attempt to purchase a tobacco product from the retailer. ${ }^{[4]}$ If the retailer fails to ask for a photographic ID and/or attempts to sell the tobacco product, the retailer fails the compliance check. When such failures (or violations) occur, the FDA takes corrective action, including issuing a warning letter, escalating civil money penalties, or issuing a No-Tobacco-Sale Order for repeated violations within a specific time period. This study analyzes results from FDA compliance-check inspections among outlets of the first regional chain retailer to adopt RACS for JUUL products (henceforth: the RACS chain). This study reports the incidence of failures observed in FDA compliance check inspections involving ENDS products and nonENDS tobacco products for the RACS chain and for other retailers in the same geographies who have not implemented RACS. Data was assessed in 2 time periods: (1) the time before the RACS chain implemented RACS, and (2) the time after the RACS chain implemented RACS. This allows for the evaluation of the potential impact of RACS on compliance check failures involving ENDS products. This study provides additional support beyond JLI's pilot study to support the finding that RACS can effectively improve retailer compliance by restricting underage access and thus reducing underage use.

\section{Analysis}

\subsection{Data and Methods}

Data was collected from the FDA's compliance check inspection database, which is publicly available and accessible through the FDA's website. ${ }^{[4]}$ These data contribute to the broader evaluation of direct access to tobacco products among those users who do not meet the legal age requirements.

The FDA's compliance check inspection database provides the following information for compliance checks resulting in failures: product category (e.g., cigarettes, ENDS, etc.) and brand information. It also provides the total number of compliance checks conducted, but not the total number of inspections segmented by product category or brand. Therefore, it is possible to discern the number of inspection failures by product category, but not the total number of inspections by product category. This study reports the total counts of age-verification failure cases for attempted purchases in the categories of ENDS and non-ENDS tobacco 
products.

This analysis is based on data collected between the second half (i.e., July 1 to December 31) of 2016 and the first half (i.e., January 1 to June 30 ) of 2020; beginning with the time point when the FDA extended its regulatory authority to all tobacco products, including e-cigarettes, cigars, hookahs and pipe tobacco, ${ }^{[1]}$ and ending in March 2020, when the FDA temporarily stopped compliance check inspections due to the outbreak of COVID-19.

The analysis focuses on the RACS chain that implemented RACS by July 29, 2019 in more than 800 stores in 12 states (AZ, GA, IA, IL, KS, MO, NC, NE, OK, OR, SC, and TX). It was applied only to JUUL products (i.e. other ENDS and tobacco products were not subject to RACS). Based on the FDA's compliance check inspection database, within the RACS chain outlets, there were 1,050 pre-implementation inspections and 217 post-implementation inspections.

We compare the number of compliance failure cases for the RACS chain stores with the number of compliance failure cases for other retailers in the same geographic regions as the RACS chain (henceforth: non-RACS retailers in the same states). To be consistent with the implementation schedule of the RACS chain, observations in 2019 were aggregated by 2 periods, the first being January 1 to July 29, 2019, and the second being July 30 to December 31, 2019.

To assess the null hypothesis that the differences in failures between the RACS chain and the other retailers could have occurred by chance alone, we conducted a bootstrap-based test. To simulate the 217 post-RACS inspections in the RACS chain, 217 observations were randomly selected from the 24,302 inspections from non-RACS retailers in the same states during the same time periods, and the number of failure cases from ENDS and non-ENDS tobacco categories were counted. That process was then repeated 100,000 times, and the likelihood for the count of ENDS failures in the RACS chain was observed. That is, if the number of failures observed in the RACS chain was lower than would have occurred by chance, this would have supported the argument that RACS reduced the number of inspection failures. The underlying assumptions were: (1) the FDA inspection would have been consistent across the RACS and non-RACS retailers in the same states during the same time periods; (2) the compliance performance of the RACS chain would have been similar to that of non-RACS retailers in the same states during the same periods if RACS would not have been implemented in the RACS chain.

\subsection{Results}




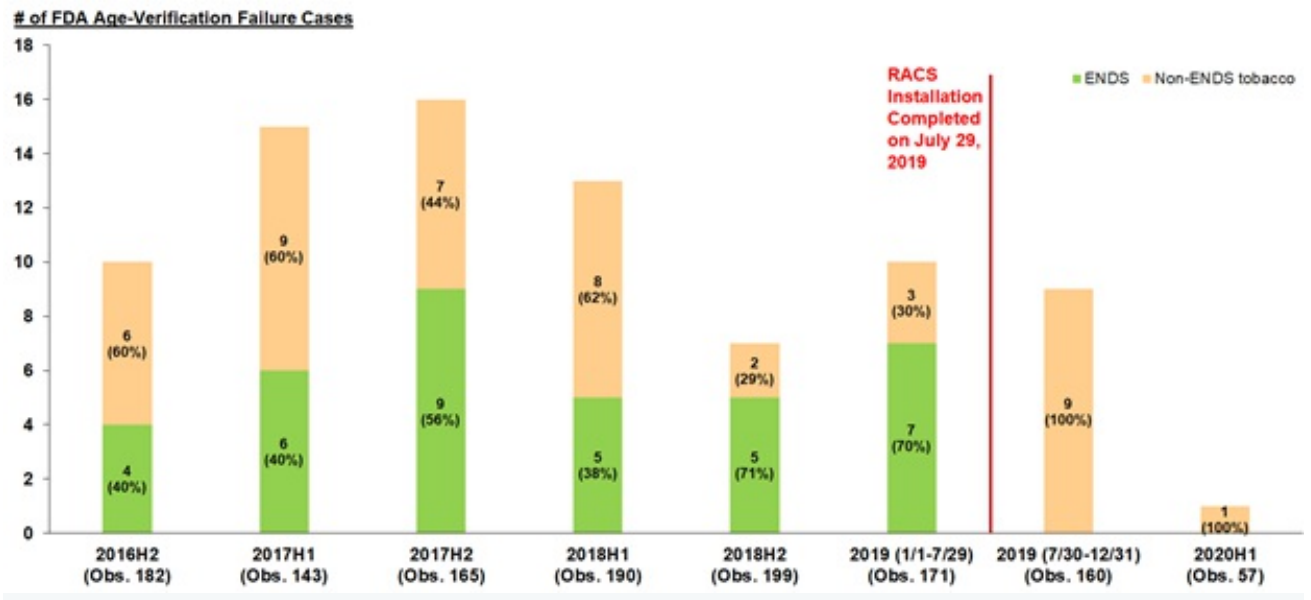

Figure 1 FDA Age-Verification Failure Cases from the RACS Chain Outlets

Figure 1 illustrates the number of inspections resulting in a failure for age-verification compliance across ENDS and non-ENDS tobacco-product categories among the RACS chain outlets before and after the implementation of RACS on July 29, 2019. Before the implementation of RACS across the RACS chain outlets, the FDA conducted 1,050 inspections and reported 71 compliance-check failures, of which 36 (50.7\%) came from inspections on ENDS. After the implementation of RACS, in the following 8 months, there were 217 inspections resulting in 10 failure cases. All failure cases involved other tobacco products; none involved ENDS products.

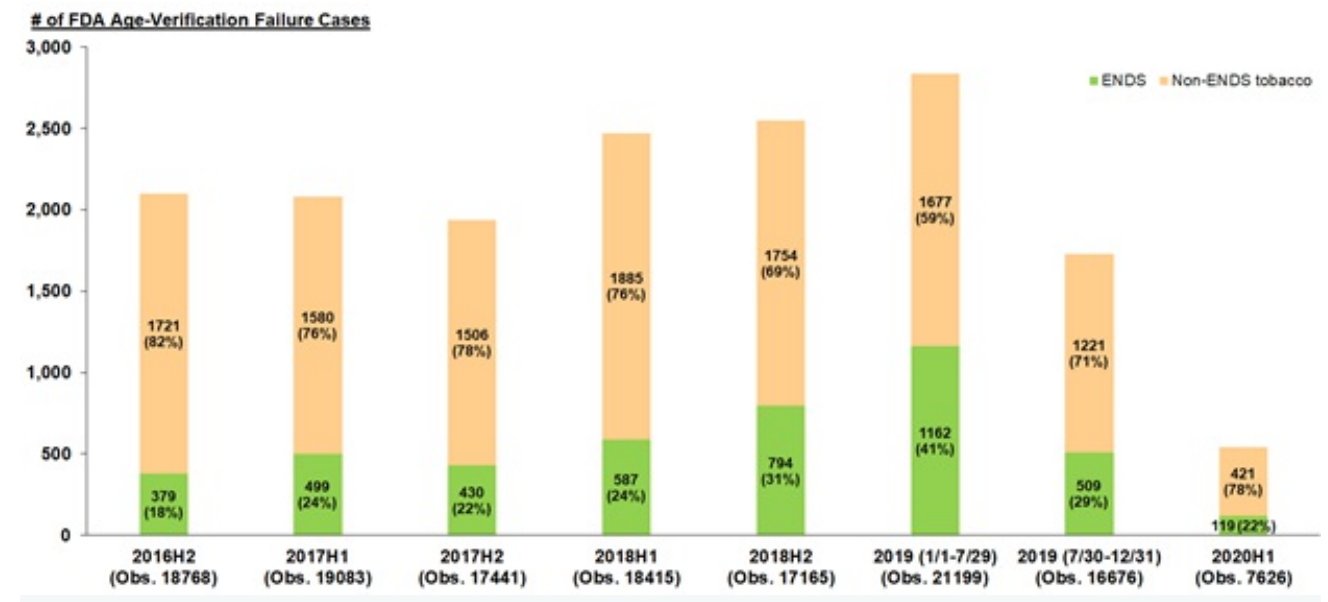

Figure 2 FDA Age-Verification Failure Cases from Non-RACS Retailers in the Same States Where the RACS Chain Stores are Located

Figure 2 illustrates the incidence of compliance check failures for non-RACS retailers in the same states. Among all failure cases from these retailers, 28\% involved ENDS between the second half of 2016 up until July 29, 2019. The incidence of compliance check failures involving ENDS was also 28\% after July 29, 2019 (i.e. the period in which the RACS chain implemented RACS). To examine the concern that the states where the RACS chain outlets are located could be different from the rest of the country in terms of FDA compliance check inspections, this study also presents the FDA compliance check failures from all tobacco retailers in the United States except the RACS chain in Appendix Figure A1, with very similar results as shown in Figure 2. 
The bootstrap simulation based on the observations of non-RACS retailers in the same states on and after July 30, 2019 showed that the probability of observing zero failure cases from ENDS - as seen in the RACS chain dataset - by chance alone was $0.3 \%$. This suggests that the zero ENDS failure rate in the RACS chain is unlikely to be a chance finding.

\section{Discussion}

In the period following implementation of RACS in the RACS chain, 217 inspections found no ageverification failures for sale of ENDS products for this retailer. In contrast, a substantial proportion of inspection failures in a geographically-matched set of non-RACS retailers were due to failure to require age verification for the sale of ENDS. Statistical analysis showed that the zero incidence of ENDS ID-verification failures in the RACS chain was unlikely to have occurred by chance alone, suggesting it was due to the implementation of RACS. This finding suggests that RACS may have been effective at reducing retailer age-verification compliance failures for ENDS products.

A previous pilot study supports that RACS is effective in improving retailer compliance with age-verification requirements for the purchase of tobacco products. ${ }^{[3]}$ The results of this analysis, based on publicly available data from the FDA's compliance-check inspections in a large and geographically diverse set of retail outlets, further support that RACS implementation is associated with fewer failures to enforce age verification for ENDS products.

This assessment based on publicly available FDA compliance check data is subject to an important limitation: the FDA does not release the total number of compliance checks by product category. Therefore, it is possible that the FDA did not conduct any ENDS-related inspections in the RACS chain after RACS implementation, thus resulting in zero ENDS-related compliance failures. However, based on the results as shown in Figure 2, it is reasonable to assume that the FDA conducted compliance check inspections at a regular cadence for all tobacco products, including ENDS, after the RACS implementation date.

The FDA reported no compliance check failures for any ENDS products in the RACS chain following RACS implementation, despite the fact that RACS was configured for JUUL products only. This could be explained by a spillover effect on non-JUUL ENDS products and contributed to the drop-in failure cases from the ENDS category. The RACS chain applied the configuration sometime after July 2019 to all ENDS products but not to non-ENDS tobacco products.

Another limitation was that the implementation of RACS was not randomly assigned, but based on the retail chain's commitment to age verification. However, were this the case, it would be expected that the rate of all compliance check failures for all tobacco products would have declined in this retail chain following RACS implementation. However, the effect was limited only to ENDS, suggesting the implementation of RACS as the cause of the change. 


\section{Conclusion}

The results from the FDA's compliance check inspections add to the evidence from prior research, indicating that automated, technologically-based standards, such as JLI's RACS, can effectively improve retailer compliance to restrict underage access and thus reduce underage use. ${ }^{[3]}$ Similar approaches should be considered for all tobacco products and potentially other age-restricted products.

\section{Abbreviations}

- AZ - Arizona

- ENDS - Electronic Nicotine Delivery System

- FDA - U.S. Food and Drug Administration

- GA - Georgia

- IA - lowa

- ID - Identification

- IL - Illinois

- JLI - JUUL Labs Inc.

- KS - Kansas

- MO - Missouri

- NC - North Carolina

- NE - Nebraska

- OK - Oklahoma

- OR - Oregon

- POS - Point of Sale

- RACS - Retail Access Control Standards

- SC - South Carolina

- TX - Texas

- U.S. - United States

\section{Appendix}




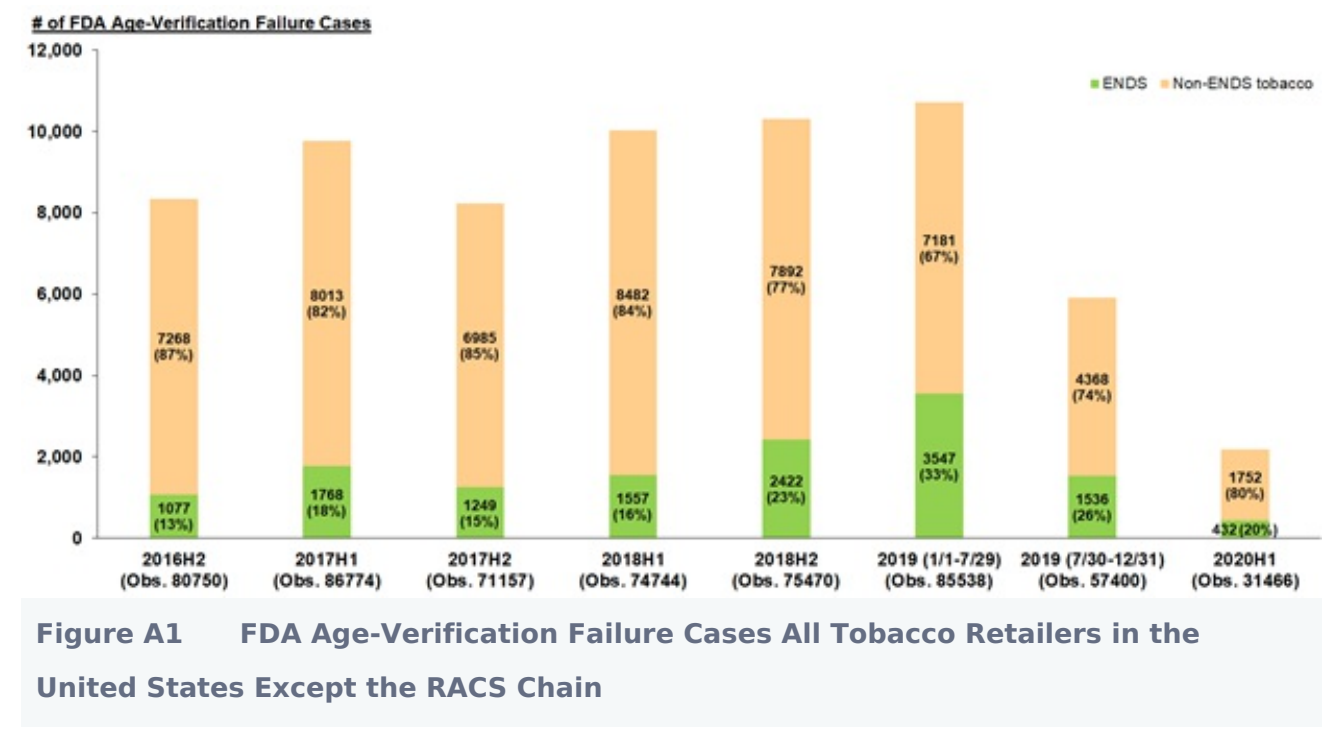

\section{References}

1. a, bUS Food and Drug Administration. (April 2020). Enforcement priorities for electronic nicotine delivery system (ENDS) and other deemed products on the market without premarket authorization. Guidance for industry. https://www.fda.gov/regulatory-information/search-fda-guidance-documents/ (Accessed 11 May 2021).

2. `JUUL Labs Inc. (2019). Retail Access Control Standards (RACS) program. https://www.racscompliance.org/documentation (Accessed 11 May 2021).

3. a, b, c, dT. Chen, S. Prakash, A. Zion, J. Joselow, S. Shiffman et al. (2021). Improving Retailer Compliance for Tobacco Purchases: Pilot Study Findings. American Journal of Health Behavior. 45(3):576-587.

4. a, b, CU.S. Food and Drug Administration, CTP Compliance \& Enforcement. (2020). Compliance Check Inspections of Tobacco Product Retailers. 